\title{
Gerenciamento de projetos: o impacto do uso dos indicadores de desempenho no resultado do projeto.
}

\author{
Francisco Ueliton Nogueira Barboza Filho, Eng. \\ Marly Monteiro de Carvalho, $\operatorname{Prof}^{\mathrm{a}} \mathrm{Dr}^{a}$. \\ marlymc@usp.br
}

\begin{abstract}
Alberto Wunderler Ramos, Prof. Dr.
O foco deste trabalho é apresentar as metodologias utilizadas no Gerenciamento de Desempenho dos Projetos e suas métricas, a ser discutido a partir de três questões principais: (I) existe diferença entre os indicadores de desempenho utilizados no gerenciamento de projetos de acordo com o setor industrial da organização executora? (II) estes indicadores são utilizados para suportar ações corretivas e evidenciar trade-off? e, (III) a utilização sistemática de indicadores tem impacto positivo nos resultados do projeto? A opção metodológica usada foi a de estudo de múltiplos casos. O estudo foi desenvolvido em 7 organizações de diferentes setores (automotivo, TI,

telecomunicações, educação e farmácia), abrangendo dados de 21 projetos. A análise estatística dos dados referentes aos projetos apresenta indícios de correlações positivas do uso de indicadores de desempenho para adoção de ações corretivas tanto no grau de atendimento aos objetivos dos clientes quanto no grau de sucesso do projeto.
\end{abstract}

Palavras-chave: gestão de projeto; desempenho; indicadores.

The focus of this work is to show the methodologies applied to the Project Performance Management and its metrics, to be discussed from three key questions: (I) is there difference between performance indicators applied in the Project Management according to the industrial segment of the organization? (II) have performance indicators of project been used to support corrective actions and to evidence trade-off?, and (III) have the project performance indicators systematic a positive impact in the project results? The methodological option used was multiple case studies. This study was enhanced by 7 organizations of different segments (automotive, information technology, telecom, education and pharmacy), collecting data from 21 projects. The statistical analysis of the projects data allowed the verification of some positive correlations among: customer objective attendance grade and the successful project grade related to the use of performance indicators for corrective action taken.

Keywords: project management; performance; indicators 


\section{Introdução}

Em tempos de competição crescente e globalização, o sucesso dos projetos se torna cada vez mais crítico para o desempenho do negócio. Não obstante, muitos projetos continuam sofrendo atrasos, ineficiências e falhas (RAZ et al, 2003). Uma pesquisa relacionada a projetos na área de tecnologia da informação (TI) aponta para uma taxa de sucesso de apenas 28\% (STANDISH GROUP, 2001).

Observa-se que a literatura sobre fatores críticos de sucesso (critical success factors - FCSs) em projeto é abrangente (PINTO; SLEVIN, 1988; PINTO; PRESCOTT, 1988; PINTO; MANTEL, 1990; BELOUT; GAUVREAU, 2004), e, de maneira geral, enfatiza as áreas de prazo, custo, escopo e qualidade como críticas. Pinto e Sleven (1987) acrescentam ainda que, um projeto é considerado um sucesso em sua implementação, caso atenda também os critérios de eficácia e satisfação do cliente. Não obstante, observase uma lacuna na literatura no que concerne ao gerenciamento do desempenho dos projetos, em geral restrita aos indicadores de análise do valor agregado (earned value analysis - EVA) e indicadores financeiros (ANBARI, 2003).

Neste contexto, o adequado gerenciamento do desempenho dos projetos é aspecto crítico para a reversão deste quadro, sendo objeto de pesquisa deste trabalho. O objetivo, apresentar as principais metodologias e indicadores utilizados para o gerenciamento do desempenho dos projetos em diferentes setores industriais, bem como analisar o impacto de seu uso sistemático no resultado dos projetos. Adicionalmente, pretendese investigar os trade offs entre os diversos FCSs.

Este artigo está estruturado em cinco seções. A seção 2 apresenta a síntese do quadro teórico, discutindo as metodologias empregadas no Gerenciamento de Desempenho dos Projetos e seus indicadores. A seção 3 apresenta a abordagem metodológica utilizada, detalhando as questões da pesquisa, os critérios de seleção dos casos e dos projetos. A seção 4 apresenta o estudo de campo realizado em 7 organizações de 4 setores (automotivo, tecnologia da informação, telecomunicações, educação e farmacêutico), bem como os resultados levantados de 21 projetos, a análise da correlação estatística das variáveis estudadas e a análise e discussão dos resultados obtidos. Finalmente, a seção 5 traz as conclusões, limitações e recomendações para trabalhos futuros.

\section{Gestão do desempenho em projetos: síntese do quadro teórico}

Nesta seção é apresentada a síntese do quadro teórico deste trabalho, que envolve os temas: de gestão de desempenho em projeto, as metodologias e as métricas utilizadas para avaliação de desempenho, destacando-se a análise do valor agregado (EVA - Earned Value Analysis) e os indicadores financeiros.

\subsection{Gestão de desempenho e sucesso em projetos}

O ponto de partida deste trabalho é a distinção entre o desempenho do produto do projeto e o desempenho do gerenciamento do projeto, pois os dois estão interligados, porém são diferentes, e é importante não confundi-los (BRYDE, 2003). Desta forma, um projeto pode ser visto como um "sucesso" apesar do Gerenciamento de Projeto falho e vice versa (DE WITT, 1988).

De uma forma tradicional, a diferença entre um alto e um baixo desempenho do projeto foi definida pelo time de projetos como atendimento ao custo, ao tempo e à qualidade, conhecido como triângulo de ferro (BRYDE; BROWN, 2004). Desde os anos 80, pesquisadores e praticantes defendem o uso de outras formar medir o desempenho dos projetos. Observa-se que um consenso tem emergido na literatura que estabelece que indicar a desempenho é mais complexo que meramente medir custo, tempo e qualidade (BRYDE; BROWN, 2004; CARVALHO et al, 2003). 
De uma forma geral, é possível medir o desempenho, monitorar e controlar os projetos confrontando o que foi planejado com o que está sendo executado (CARVALHO; RABECHINI JR., 2005). No entanto, estes autores alertam que não é suficiente comparar a planejado com o realizado, pois é necessário olhar em uma perspectiva maior de longo prazo e alinhada às estratégias organizacionais. Desta forma, em uma organização, a escolha de quais indicadores medir e monitorar estarão vinculados a uma decisão anterior com relação à estratégia de competição da empresa e dos Fatores Críticos de Sucesso, alinhados à estratégia selecionada (MUSCAT ; FLEURY, 2000). Shenhar (2004) também destaca a dimensão estratégica, pois afirma que gerenciar apenas prazo, custo e escopo conforme planejado não é suficiente para a garantia de sucesso no projeto, e apresenta a abordagem SPL (Strategic Project Leadership), composta de cinco elementos: estratégia, organização, ferramentas, processos e espírito.

Além dos sistemas de indicadores de desempenho do projeto, vários pesquisadores publicam trabalhos que tentam correlacionar os impactos positivos ou negativos de abordagens, técnicas e metodologias no desempenho dos projetos. Entre elas pode-se destacar o impacto do Escritório de Projetos (PMO) citado por Dai (2004), no qual em pesquisa realizada conclui que empresas que tinham o Escritório de Projetos apresentavam melhores desempenhos nos projetos em relação a empresas que não possuíam. Patah e Wells, (2005) afirmam que a seleção da correta estrutura organizacional pelas empresas será uma questão estratégica para o sucesso de seus projetos. Pitsis et al (2003) comentam os excelentes resultados obtidos na utilização da abordagem de "Futuro Perfeito" no projeto da preparação da cidade para as Olimpíadas de Sidney. Os resultados da pesquisa feita por Sarin eMahajam, (2001) demonstram a relação entre a avaliação do desempenho individual dos membros do time do projeto, e aplicação de diferentes tipos de reconhecimentos ou premiações com o aumento da satisfação geral da equipe, e por conseqüência, o efeito positivo no desempenho do projeto. Tamhain (2004) conclui que condições organizacionais que satisfazem necessidades pessoais e profissionais dos membros do time do projeto geram um forte efeito no comprometimento dos mesmos, fazendo com que barreiras sejam mais facilmente ultrapassadas e que o desempenho do time e conseqüentemente do projeto sejam melhores. Raz et al (2002) apresentam as relações entre os tipos de projetos e a aplicação de práticas de gerenciamento de riscos e como estas práticas contribuem no sucesso (desempenho) do projeto.

Outra questão que merece destaque é a temporal. Bryde e Brown (2004) sustentam que a medição deve incorporar critérios que considerem o projeto além da entrega, poderia ser incluído o atendimento de objetivos financeiros de longo prazo como a taxa interna de retorno. Carvalho e Rabechini Jr (2005) destacam ainda a retenção de clientes como um indicador de sucesso no longo prazo.

Um ponto muito importante para as organizações é o estabelecimento de procedimentos e sistemas de medição que não apenas coletem informações relevantes de forma operacional, mas que isso seja devidamente comunicado aos interessados de forma a influenciar o comportamento deles na direção do negócio (WETTER, 2001 apud HIKAGE et AL, 2006). Weber e Schaffer (2001) corroboram desta visão argumentando que os sistemas de medição de desempenho estão sendo usados como prática corporativa por um longo período, porém, o seu valor como ferramenta de gerenciamento não está difundido, essencialmente devido ao costume de estarem dirigidos a dados existentes com poucas informações úteis.

Em síntese, existem muitas técnicas para monitoramento e controle dos resultados do projeto, contudo, diversos autores destacam a análise do valor agregado (EVA- Earned Value Analysis), que também é conhecida como gerenciamento de valor agregado (EVM - Earned Value Management), e a utilização de indicadores financeiros, como ferramentas eficazes para monitorar o desempenho do projeto, com ênfase nos parâmetros financeiros, prazo e custo (GRANOT, 1997, ANBARI 2003; PMI, 2004, CARVALHO; RABECHINI JR, 2005). Nas próximas seções serão abordadas algumas destas técnicas que se julgou relevante no contexto desta pesquisa. 


\subsection{Análise de valor agregado}

Existem muitas técnicas para monitoramento e controle dos resultados do projeto, contudo, diversos autores destacam a análise do valor agregado (EVA- Earned Value Analysis), também conhecida como gerenciamento de valor agregado (EVM - Earned Value Management), e a utilização de indicadores financeiros, como ferramentas eficazes para monitorar o desempenho do projeto, com ênfase nos parâmetros financeiros, prazo e custo (GRANOT, 1997, ANBARI 2003; CARVALHO; RABECHINI JR, 2005). Nesta seção, serão abordados alguns indicadores de desempenho do projeto utilizados neste método.

Uma das técnicas mais utilizadas para mensuração e monitoramento do desempenho do projeto é o gerenciamento do valor agregado (EVM), que compara o valor do trabalho efetivamente realizado ao montante originalmente estimado no orçamento, integrando para tal as áreas de escopo, custo e prazo (PMI, 2004).

O referencial para o EVM é a linha base (baseline) do projeto, que reflete as estimativas planejadas para o orçamento do projeto. As análises são feitas através dos desvios ocorridos ao longo do projeto em sua baseline (Carvalho; Rabechini Jr, 2005). A EVA utiliza três medidas para o cálculo dos índices e desvios no desempenho de prazo e custo do projeto, quais sejam: Planed Value - PV (Valor Planejado ou Orçado); Actual Cost - AC (Custo Real) e o Earned Value - EV (Valor Agregado). Estas medidas podem ser definidas da seguinte forma:
"O valor planejado ou orçado (PV) é o valor total orçado para ser gasto em uma determinada atividade, pacote ou projeto, em um determinado ponto de verificação do projeto... O AC é o custo real, aquele desembolsado pela empresa no período...O valor agregado (EV) é o montante orçado para o trabalho efetivamente realizado no período. Para determinar este valor é preciso conhecer qual foi a porcentagem do trabalho planejado para o período que foi de fato executada...” (CARVALHO; RABECHINI Jr, 2005).

Com base nestas três medidas, é possível calcular os indicadores-chave da EVA, que são: variação de custo (Cost Variance - CV) e o índice de desempenho em custo (Cost Performance Index - CPI); e a variação de prazo (Schedule Variance - SV) e o índice de desempenho em prazo (Schedule Performance Index - SPI). Estes índices podem ser calculados segundo Anbari (2003) da seguinte forma:

- Os dois indicadores de custo analisam o valor orçado para o trabalho efetivamente realizado/agregado (EV) face ao valor efetivamente desembolsado (AC), sendo calculados da seguinte forma:

o variação de custo: $\mathrm{CV}=\mathrm{EV}-\mathrm{AC}$; e

o índice de desempenho em custo: $\mathrm{CPI}=\mathrm{EV} / \mathrm{AC}$.

- Os dois indicadores de prazo analisam o valor orçado para o trabalho efetivamente realizado/agregado (EV) face ao valor orçado para o trabalho que deveria ser feito do período de análise (PV), sendo calculados da seguinte forma:

o variação de prazo: $\mathrm{SV}=\mathrm{EV}-\mathrm{PV}$;

$\mathrm{o}$ índice de desempenho do prazo: $\mathrm{SPI}=\mathrm{EV} / \mathrm{PV}$

Observando-se as fórmulas dos índices CPI e SPI, verifica-se que quanto mais próximo 1.0, significa que o custo e o prazo do projeto ocorreram conforme o planejado na baseline do projeto (CARVALHO; RABECHINI JR, 2005). A Figura 1 ilustra os indicadores apresentados para a análise de Valor Agregado (EVA). 


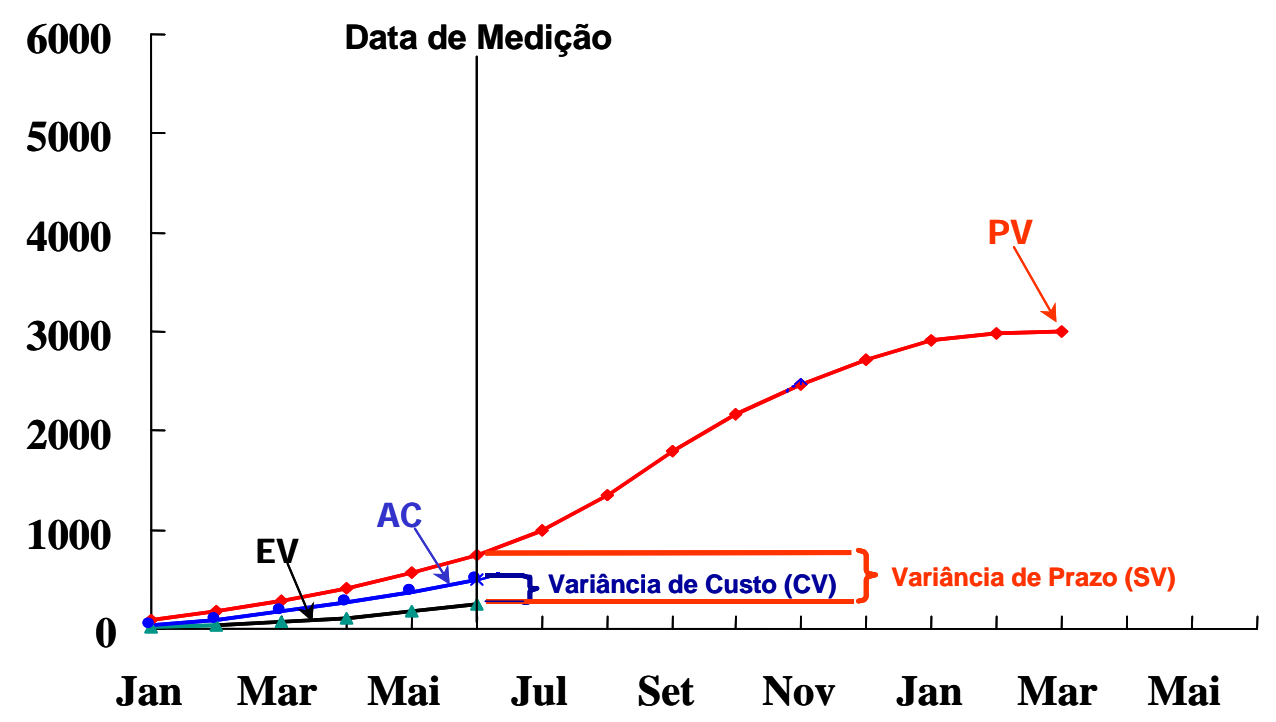

Figura 1 Análise do valor agregado (CARVALHO; RABECHINI JR, 2005)

Anbari (2003) corrobora desta análise ressaltando que quando o resultado é o mais próximo de 1,00 possível, significa que o desempenho é eficiente e está dentro das metas estabelecidas, conforme ilustra a Figura 2.

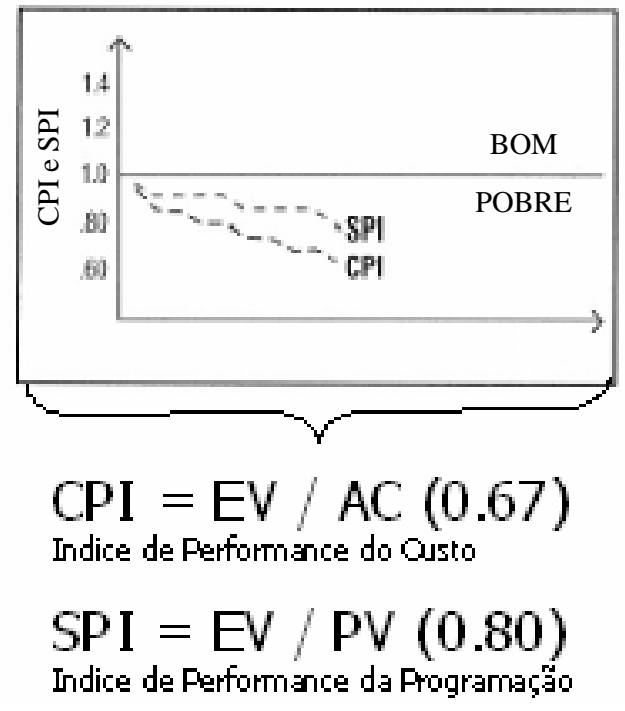

Figura 2 - Gráfico TEAC (ANBARI, 2003).

Segundo Carvalho e Rabechini Jr (2005), com base nesta analise podem ser classificadas quatro categorias de projetos: Mal Gerenciado, Rápido \& Gastão, Lento \& Econômico e Bem Gerenciado. "O projeto mal gerenciado é aquele que teve desempenho negativo tanto em prazo (SV negativa) como em custo (CV negativa) e, portanto, obtive índices inferiores a 1,0, tanto para CPI como para SPI. O projeto Rápido \& Gastão é aquele que teve um bom desempenho em prazo (SV positiva), mas para isto comprometeu seu desempenho em custo (CV negativa) e, portanto, obtiveram índices inferiores a 1,0 para CPI, mas 
superiores a 1,0 para SPI. O projeto Lento \& Econômico é aquele que, ao contrário da categoria anterior, teve um bom desempenho em custo (CV positiva e CPI maior que 1,0), mas para isto comprometeu seu desempenho em prazo (SV negativa e SPI menor que 1,0). Finalmente, o projeto bem gerenciado é aquele em que o desempenho tanto em prazo como em custo superou positivamente o planejado"

\subsection{Indicadores Financeiros}

Na seqüência abordam-se alguns indicadores financeiros comumente utilizados no Gerenciamento de Desempenho de Projetos. O VPL (Valor Presente Líquido) também conhecido por NPV (Net Present Value), é considerado como um poderoso instrumento para avaliação financeira, porque incorpora fatores relevantes de valor tais como: fluxos de caixa futuros e custo de capital (COPELAND et al., 1996). O VPL considera não só o valor dos ativos atuais, mas também o valor dos fluxos futuros como aqueles derivados dos investimentos existentes e dos projetos a serem realizados.

Também na linha financeira, destaca-se o ROA (Return On Assets ou Retorno sobre os Ativos) e o ROS (Return On Sales ou Retorno sobre Vendas); para Fachini (2005) o ROA indica se a organização faz bom uso do seu ativo total para gerar retornos, medindo o seu poder de ganho por unidade monetária investida (ROA = (Resultado Total / Ativo Total) x 100); de acordo com definições do "The Free Dictionary" o termo ROS trata-se de uma medida útil ao gerenciamento, fornecendo direções do lucro que está sendo obtido por dólar vendido (ROS = Lucro Líquido Antes de Impostos / Vendas).

Com relação ao prazo, além do acompanhamento de cronogramas, fases dos projetos, milestones (eventos chaves ou marcos temporais do projeto), e outras renomadas ferramentas; pode-se observar que alguns indicadores simplistas para o acompanhamento de atividades são amplamente utilizados tais como: \% de atividades entregues no tempo esperado (\% atividades entregues no tempo esperado $=$ numero de atividades que atenderam o prazo estipulado / total de atividades), \% de atividades atrasadas (\% de atividades atrasadas $=$ número de atividades atrasadas / total de atividades).

\section{Método}

Conforme apresentado na seção introdutória deste artigo, o objetivo central deste trabalho é discutir o impacto do uso de metodologias e de indicadores de desempenho no resultado dos projetos. Com base no quadro referencial teórico, foram extraídas três questões principais, que balizaram a pesquisa de campo:

(I) existe diferença entre os indicadores de desempenho utilizados no gerenciamento de projetos de acordo com o setor industrial da organização executora?

(II) os indicadores de desempenho de projetos são utilizados para suportar ações corretivas e evidenciar trade-off?, e

(III) a utilização sistemática de indicadores de desempenho de projetos tem impacto positivo nos resultados do projeto?

A opção metodológica usada foi a de estudo de múltiplos casos, cujos critérios de seleção foram: diversidade de setores industriais e importância da área de gestão de projetos. Para fazer um estudo longitudinal nestas organizações, optou-se pelo levantamento de dados documentais e entrevistar envolvidos em projetos recentes, destas organizações (YIN, 1981).

Com base nestes critérios, foram selecionadas 7 organizações dos seguintes setores: automotivo, tecnologia da informação, telecomunicações, educação e farmacêutico. Além disto, foram coletados dados de 21 projetos, que forneceram um panorama abrangente no que concerne a: incerteza tecnológica; orçamentos (projetos de milhares de dólares até acima de 3 milhões de dólares) e resultados (casos de sucesso e fracassos). 
O desafio deste trabalho foi desenvolver um instrumento de pesquisa que permitisse a coleta de dados base, para iniciar uma análise de correlação entre: os fatores (graus), indicadores utilizados, segmentos das organizações e se ações corretivas foram adotadas; para então discutir os resultados com base nos objetivos desta pesquisa e visualizar "indícios” de respostas associadas às questões da pesquisa, apresentadas no início desta seção. O instrumento de pesquisa elaborado apresentava questões abertas e fechadas. O Anexo deste artigo apresenta as questões fechadas deste instrumento, que era dividido em dois blocos: dados de referência e dados do projeto. O primeiro bloco apresentava os dados de referência utilizados para caracterização do entrevistado e da empresa. O segundo bloco apresentava questões para a caracterização dos projetos, sobre o uso de indicadores de desempenho em projetos e sobre os resultados do projeto.

Os projetos podem ser caracterizados da seguinte forma:

- A estratificação dos projetos por segmento é a seguinte: $47 \%$ automotivo, $28 \%$ tecnologia da informação, 14\% telecomunicações, 5\% farmacêutico, e 5\% educação;

- A maioria dos projetos (62\%) dispunha de um Gerente de Projeto dedicado;

- Dentre as organizações estudadas, $45 \%$ realizaram entre 11 e 40 projetos nos últimos 2 anos; $40 \%$ realizaram entre 1 e 10 projetos e, apenas $15 \%$, realizaram entre 40 e 100 projetos.

- Dentre os 21 projetos analisados, 55\% apresentaram orçamento entre 500 mil e 3 milhões de dólares, 22\% tinham orçamento de até 10 mil dólares, 17\% com orçamento maior que 3 milhões de dólares e apenas $6 \%$ dos projetos tinha orçamento na faixa de entre 100 mil e a 500 mil dólares.

A pesquisa considerou para a coleta de dados apenas os projetos encerrados recentemente, menos de dois anos, com objetivo de avaliar o comportamento e a interação das seguintes variáveis: duração do projeto (anos), quantidade de integrantes do time do projeto, grau de incerteza tecnológica, grau de atendimento ao escopo, grau de atendimento ao orçamento, grau de atendimento ao prazo, grau de atendimento aos objetivos do cliente final, grau de atendimento aos objetivos dos stakeholders, grau de importância do projeto para organização, considerando competitividade, e por fim grau de sucesso do projeto. A Tabela 1 apresenta os dados relativos a estas variáveis.

Tabela 1 Estatística descritiva do comportamento das variáveis da pesquisa

\begin{tabular}{llrrrr}
\hline Código & \multicolumn{1}{c}{ Variável } & N & Média & $\begin{array}{l}\text { Desv. } \\
\text { Pad. }\end{array}$ & Mediana \\
\hline & Intervalo Duração do Projeto & 20 & 1,255 & 0,79 & 1,2 \\
\hline & Qtde Integrantes do Projeto & 21 & 22,48 & 22,81 & 15 \\
\hline G.Inc. & Grau Inc. Tecnológica & 21 & 4,143 & 2,287 & 4 \\
\hline G.A.Es. & Grau Atend. Escopo & 21 & 8,333 & 1,798 & 9 \\
\hline G.A.Or. & Grau Atend. Orçamento & 21 & 7,619 & 1,987 & 8 \\
\hline G.A.Pr. & Grau Atend. Prazo & 21 & 8,048 & 2,376 & 9 \\
\hline G.A.Cl. & Grau Atend. Obj. Cliente & 21 & 7,571 & 1,859 & 8 \\
\hline G.A.St. & Grau Atend. Obj. Stakeholders & 21 & 7,952 & 1,627 & 8 \\
\hline G.Imp. & Grau Importância Projeto & 21 & 8,619 & 1,717 & 9 \\
\hline G.Sucesso & Grau Sucesso Projeto & 21 & 7,905 & 2,166 & 9 \\
\hline
\end{tabular}

A análise estatística dos dados coletados foi feita utilizando-se o software Minitab.

O desafio deste trabalho foi desenvolver um instrumento de pesquisa que permitisse a coleta de dados base, para iniciar uma análise de correlação entre: os fatores (graus), indicadores utilizados, segmentos das organizações e se ações corretivas foram adotadas; para então discutir os resultados com base nos objetivos desta pesquisa e visualizar "indícios” de respostas associadas às questões da pesquisa apresentadas no início desta seção. 


\section{Análise da pesquisa de campo}

Nesta seção, são apresentados os resultados obtidos na análise dos 21 projetos dos 7 casos estudados. Em linha com os objetivos deste trabalho, primeiramente será analisada a correlação entre as variáveis da pesquisa, apresentadas na seção anterior Tabela 1.

Numa análise preliminar para testar a coerência dos dados coletados é possível identificar correlações significativas entre as variáveis, que estão em destaque destacadas na Figura 3. Portanto, merecem destaque a correlação entre: (i) o grau de atendimento ao prazo (G.A.Pr.) e o grau de atendimento ao escopo (G.A.Es.); (ii) o grau de atendimento ao prazo (G.A.Pr.) e o grau de atendimento ao orçamento (G.A.Or.); iii) o grau de atendimento aos objetivos dos clientes (G.A.Cl.) e o grau de atendimento ao escopo (G.A.Es.); iv) o grau de atendimento aos objetivos dos stakeholders (G.A.St.) e o grau de aos objetivos dos clientes (G.A.Cl.).

\begin{tabular}{|c|c|c|c|c|c|}
\hline \multicolumn{5}{|l|}{ Correlações } & \multirow{6}{*}{ 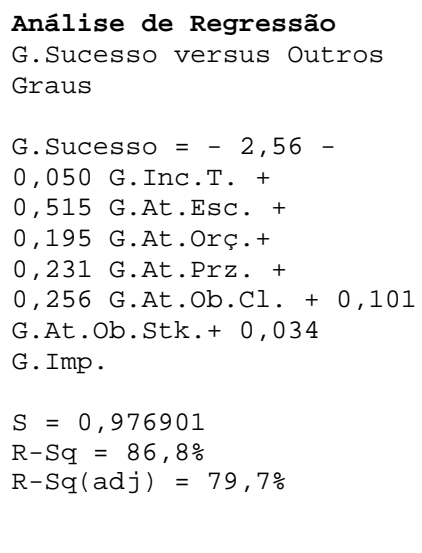 } \\
\hline $\begin{array}{rr}\text { G.A.ESC. } & -0,195 \\
& 0,398\end{array}$ & & & & & \\
\hline $\begin{array}{r}-0,219 \\
0,341\end{array}$ & $\begin{array}{l}0,359 \\
0,110\end{array}$ & & & & \\
\hline $\begin{array}{rr}\text { G.A.Prz. } & -0,121 \\
& 0,602\end{array}$ & $\begin{array}{l}\odot, 558 \\
\odot, 009\end{array}$ & $\begin{array}{l}0,534 \\
\odot, 013^{*}\end{array}$ & & & \\
\hline $\begin{array}{r}\text { G. A. Ob.St. }-\odot, 213 \\
\odot, 354\end{array}$ & $\begin{array}{l}0,330 \\
0,144\end{array}$ & $\begin{array}{l}0,334 \\
0,138\end{array}$ & $\begin{array}{l}0,259 \\
\odot, 257\end{array}$ & $\begin{array}{l}\odot, 456 \\
\odot, 038^{*}\end{array}$ & \\
\hline $\begin{array}{ll}\text { G. Imp. } & 0,193 \\
& 0,402\end{array}$ & $\begin{array}{l}0,238 \\
0,300\end{array}$ & $\begin{array}{r}-\odot, 089 \\
\odot, 702\end{array}$ & $\begin{array}{l}\odot, 127 \\
\odot, 583\end{array}$ & $\begin{array}{l}\odot, 463 \\
0,034 *\end{array}$ & \\
\hline
\end{tabular}

Nota: $\left({ }^{*} \mathrm{p}<0,05\right)$

Figura 3 Correlações \& Regressão entre as variáveis (Minitab Software)

Pode-se observar ainda na Figura 3 que o grau de sucesso correlacionou-se com o grau de atendimento: ao escopo (G.A.Es.), ao orçamento (G.A.Or.), ao prazo (G.A.Pr.), aos objetivos dos clientes (G.A.Cl.) e aos objetivos dos stakeholders (G.A.St.). Já o Grau Importância Projeto (G.Imp.) correlacionou-se apenas com o grau de atendimento aos objetivos dos clientes (G.A.Cl.)

O próximo passo foi à análise da utilização de diferentes indicadores nos diferentes setores industriais das empresas respondentes. A Figura 4 apresenta o percentual de utilização de diferentes indicadores de desempenho de projetos por diferentes segmentos. 


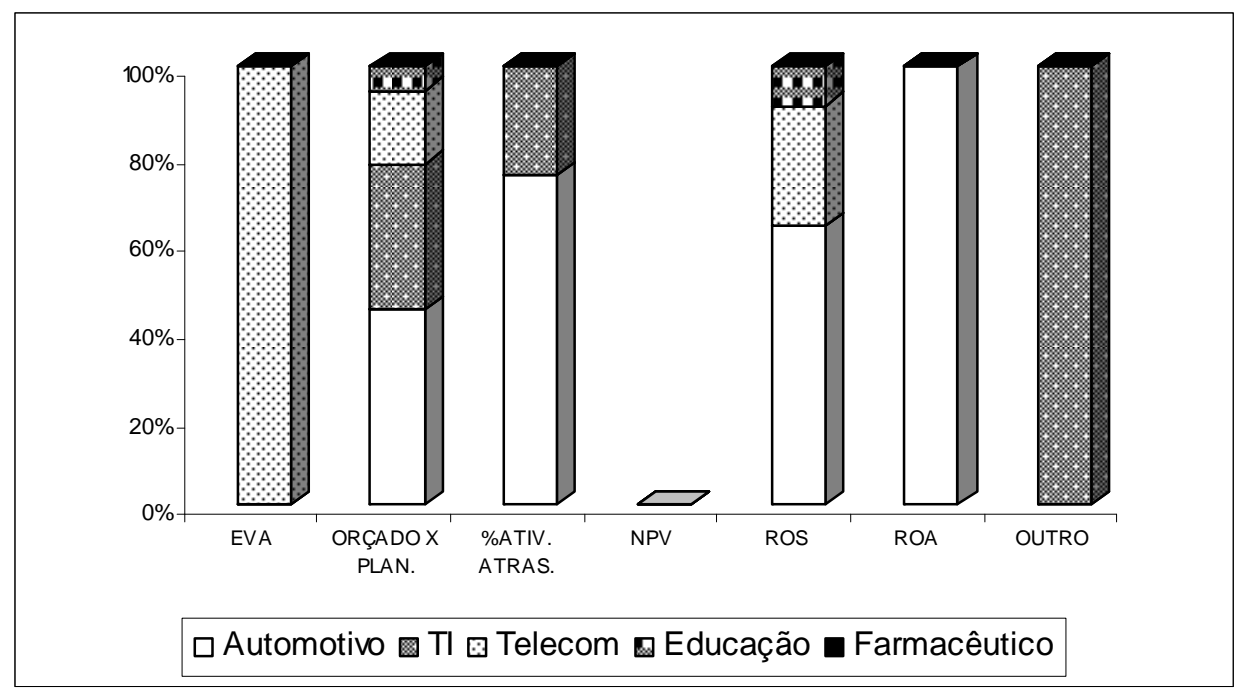

Figura 4 Porcentagem de uso de indicadores de desempenho por segmento.

Um dado que precisa ser destacado e certamente requer maiores estudos com relação ao percentual de utilização de indicadores de desempenho nos projetos, pois apenas $52 \%$ dos projetos que compõem a amostragem utilizaram algum tipo de indicador de desempenho. Destes 52\% que utilizaram algum tipo de indicador de desempenho, a grande concentração ficou no segmento automotivo. Considerando que a amostra desta pesquisa é composta pela maioria de projetos do segmento automotivo (dos 21 casos estudados, 10 projetos são do setor automotivo), desta forma, a análise pode estar enviesada, e, requer maiores ponderações e continuidade do estudo.

Observa-se na Figura 4, uma variação muito grande, com relação à utilização de alguns indicadores entre os diferentes segmentos das empresas respondentes. Outro dado a destacar nesta análise é o fato de nenhum dos respondentes ter relatado o uso do NPV ou VPL (Valor Presente Líquido) nos projetos referenciados. A análise de valor agregado (EVA) foi utilizada em apenas 3 projetos do segmento de telecomunicações, não sendo relatado em nenhum outro setor. Já o indicador financeiro ROS foi utilizado em 11 projetos, sendo que o indicador ROA foi utilizado apenas nos 7 projetos do setor automotivo. Partindo para análise sobre a aplicação destes indicadores para suportar a adoção de ações corretivas, observou-se que $52 \%$ utilizaram indicadores de desempenho para suportar adoção de ações corretivas nos projetos. Estratificando-se este dado por setor tem-se: 73\% automotivo, 18\% TI, 9\% educação, 0\% telecomunicações e $0 \%$ farmacêutico.

A Figura 5 apresenta a análise feita no software Minitab para verificar se existe diferença significativa entre o grau de atendimento do projeto aos objetivos dos clientes, considerando-se os projetos que utilizaram algum tipo de indicador de desempenho para suportar a adoção de ações corretivas (Sim) daqueles projetos que não aplicaram (Não). Nesta análise não é possível observar diferença significante da categoria SIM em relação a categoria NÃO, tanto para as variâncias como para as médias. Portanto, não há indício de que o grau de atendimento do projeto aos objetivos dos clientes esteja relacionado à utilização de indicadores de desempenho.. 


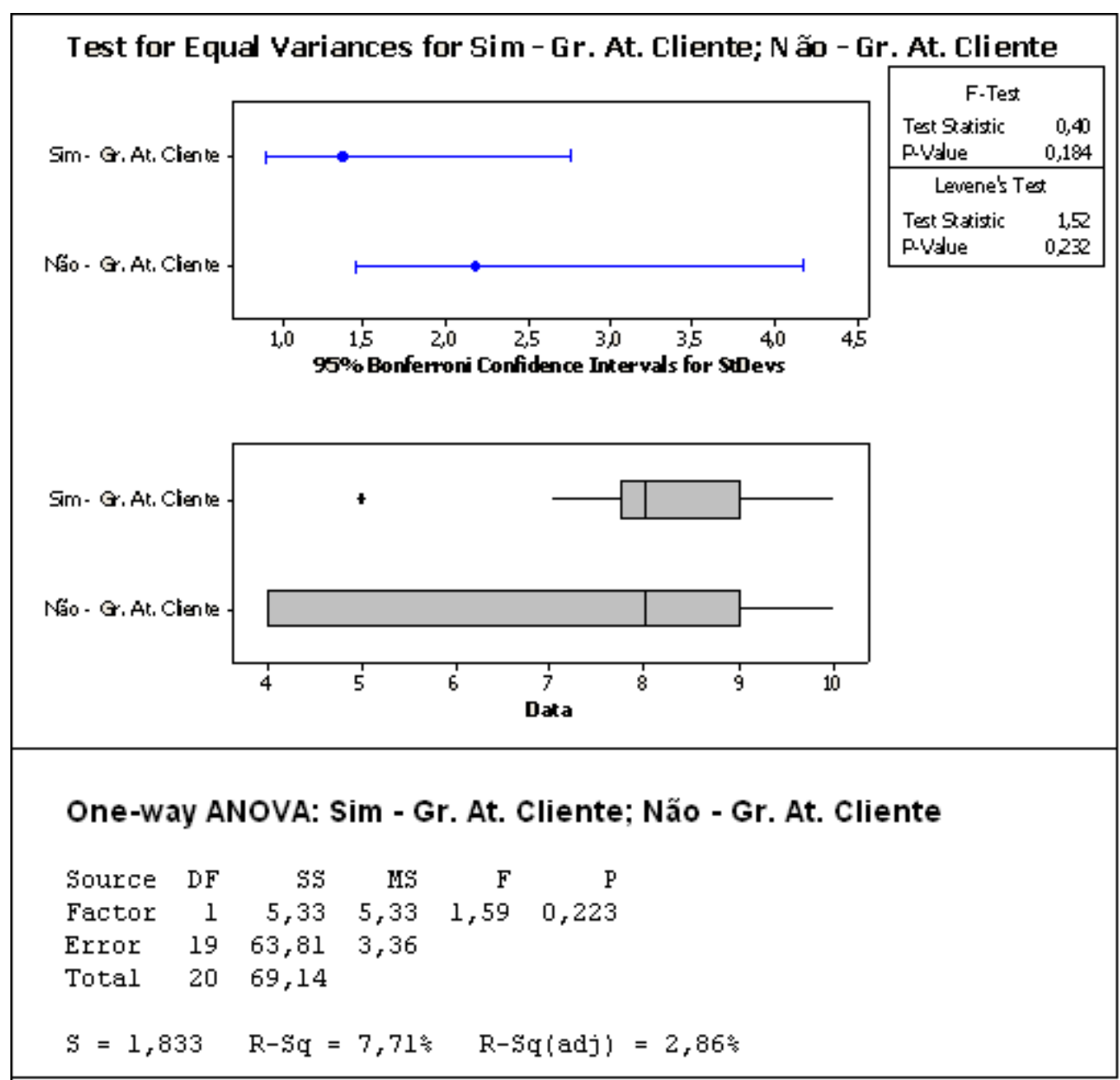

Nota: projetos (Sim) aplicaram os indicadores de desempenho para suportar a adoção de ações corretivas, e os projetos (Não) que não aplicaram.

Figura 5 Grau de atendimento do projeto aos objetivos dos clientes (Minitab Software).

A Figura 6 apresenta a análise feita no software Minitab para verificar se existe diferença significativa entre o grau de atendimento do projeto aos objetivos dos stakeholders considerando-se os projetos que utilizaram algum tipo de indicador de desempenho para suportar a adoção de ações corretivas (Sim) daqueles projetos que não aplicaram (Não). Nesta análise também não foi possível verificar diferenças significativas nem nas variâncias, nem nas médias. Portanto, não há indício de que o grau de atendimento aos objetivos dos stakeholders esteja relacionado à utilização de indicadores de desempenho. 


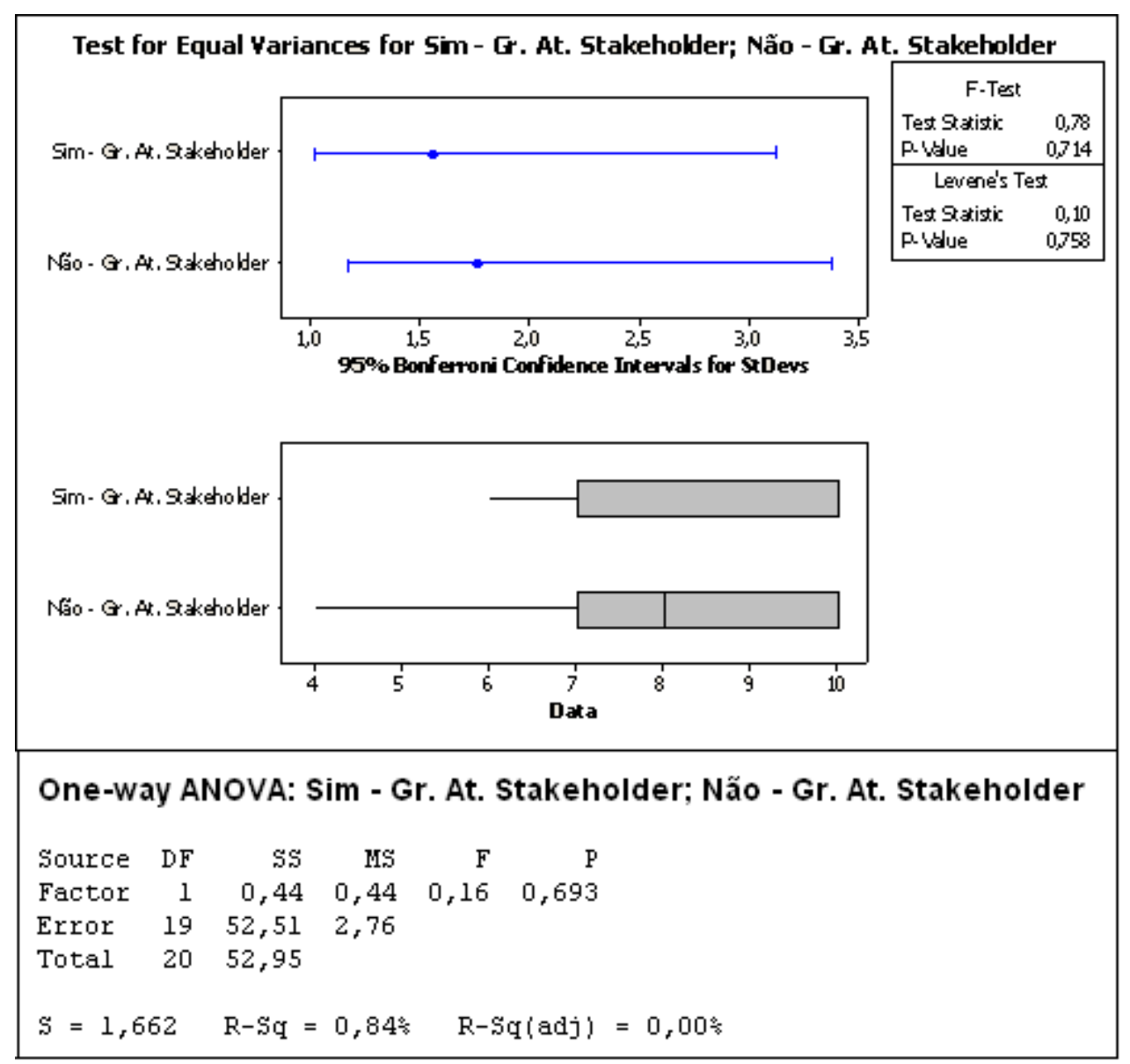

Nota: projetos (Sim) aplicaram os indicadores de desempenho para suportar a adoção de ações corretivas, e os projetos (Não) que não aplicaram.

Figura 6 Grau de atendimento do projeto aos objetivos dos “stakeholders” (Minitab Software).

A Figura 7 apresenta a análise feita no software Minitab para verificar se existe diferença significativa entre o grau de sucesso, considerando-se os projetos que utilizaram algum tipo de indicador de desempenho para suportar a adoção de ações corretivas (Sim) daqueles projetos que não aplicaram (Não). Nesta última análise também não foi possível verificar diferenças significativas para as duas categorias, nem para as variâncias nem para as médias. Portanto, não há indício de que o grau de sucesso do projeto esteja relacionado à utilização de indicadores de desempenho. 


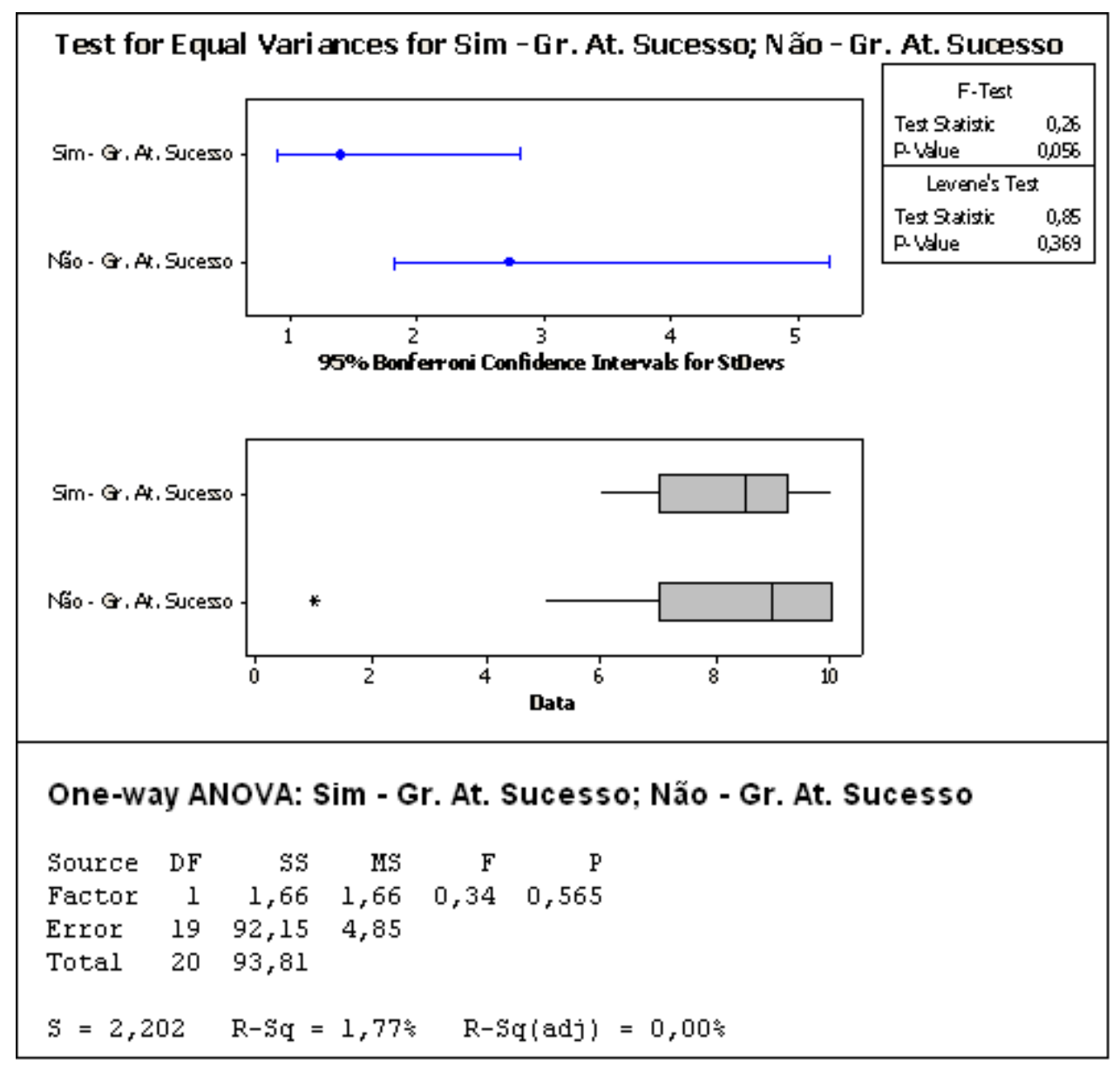

Nota: projetos (Sim) aplicaram os indicadores de desempenho para suportar a adoção de ações corretivas, e os projetos (Não) que não aplicaram.

Figura 7 Grau de sucesso do projeto (Minitab Software).

\section{Conclusões}

Destaca-se como contribuição deste artigo a revisão de conceitos relacionados aos indicadores de desempenho de projeto, em âmbito operacional e de eficiência, através da abordagem de algumas metodologias e ferramentas disponíveis na literatura. Além do quadro teórico, o trabalho apresenta dados de 21 projetos de diferentes setores, que possibilitaram a constatação com relação às questões colocadas na pesquisa.

No que concerne à diferença entre os indicadores de desempenho utilizados no gerenciamento de projetos de acordo com o setor industrial da organização executora, observou-se que os indicadores financeiros só foram utilizados nos segmentos de telecomunicações e automotivo. Já a EVA foi utilizada apenas nos projetos de telecomunicações.

Na investigação da relação entre a uso de indicadores de desempenho e o atendimento às necessidades dos clientes, stakeholders e ao grau de sucesso do projeto, verificou-se que não há indícios de impacto do uso de indicadores. 
O estudo também trouxe evidências de que o uso de indicadores de desempenho em projetos, ainda não é uma prática sistemática nas organizações, tendo sido observado em apenas a metade dos projetos estudados (52\%), embora no setor automotivo esta porcentagem seja mais expressiva atingindo $73 \%$. No que concerne aos indicadores, observa-se que o uso na EVA ainda é muito restrito tendo sido aplicado em apenas três projetos. Os indicares financeiros e os mais simples em porcentagens são os mais utilizados nas organizações estudadas.

Contudo, é importante enfatizar que existem fatores limitantes nesse trabalho tal como amostragem reduzida, o que podem distorcer e/ou comprometer os resultados finais. Além disto, a variação dos dados obtidos, dada as características dos entrevistados, que podem ter diferentes percepções de acordo com o grau de conhecimento na área de gestão de projeto, acesso às informações e experiência na utilização das ferramentas e técnicas de gerenciamento de projetos.

Portanto, para extensão destes resultados, são necessários estudos futuros de forma a dirimir as limitações aqui apresentadas, através de estudos mais detalhados e com maior representatividade estatística, bem como melhorias no instrumento de pesquisa deste trabalho, que permitirão um melhor aproveitamento das análises e melhores conclusões sobre o tema. Para continuação de pesquisa nesta área, outras questões como a importância e influência do "controle", no gerenciamento dos indicadores de desempenho de projetos, poderiam ser abordadas e contempladas no instrumento de pesquisa para análises e discussões complementares.

\section{Referências}

ANBARI, F. T. Earned Value Project Management Method and Extensions. Project Management Journal, p. 12-23, 2003.

BELOUT, A.; GAUVREAU, C. Factors influencing project success: the impact of human resource management. International Journal of Project Management 22 1-11, 2004.

BRYDE, D.J.; BROWN, D. The influence of a project performance measurement system on the success of a contract for maintaining motorways and trunk roads. Project Management Journal; Dec 2004; v. 35, n. 4; p. 57-65.

BRYDE, D.J. Modelling project management performance. International Journal of Quality \& Reliability Management, V. 20 N. 2, pp. 229-254, 2003

CARVALHO, M. M.; LAURINDO, F. J. B.; PESSÔA, M. S. P. Information Technology Project management to achieve efficiency in Brazilian Companies. In: KAMEL, Sherif. (Org.). Managing Globally with Information Technology, Hershey, p. 260-271, 2003.

CARVALHO, M. M.; LAURINDO, F.J.B. Linking strategy with a network of performance indicators: a brazilian research centre. International Journal of Business Performance Management. v. 5, n. 4, p. 285301, 2003.

CARVALHO, M. M.; RABECHINI JR. Construindo Competências para Gerenciar Projetos: Teorias e Casos. São Paulo: Atlas, 2005, 317 p.

COPELAND,T.E.; KOLLER, T.; MURRIN, J. Valuation: measuring and managing the value of companies. New York: Wiley \& Sons, 1996.

DAI, C. X.; WELLS, W. G. An exploration of project management office features and their relationship 
to project desempenho. International Journal of Project Management, pp. 523-532, 2004.

DE WITT, A. Measurement of project management success, International Journal of Project Management, Vol. 6 No. 3, pp. 164-70, 1988.

ENGWALL, M. No project is an island: linking projects to history and context. Management Science, p.789-808, 2002.

FACHINI, C. Sustentabilidade Financeira e Custos de Transição em uma organização de microcrédito no Brasil. Escola Superior de Agricultura Luiz de Queiroz, 131 p, 2005.

GRANOT, M. A Pratical Approach to Project Control. Proceedings of the 28th Annual Project Management Institute Seminars \& Symposium, p. 1012-1015, 1997.

GRANT, K. P.; PENNYPACKER, J. S. Project Management Maturity: An Assessment of Project Management Capabilities Among and Between Selected Industries. IEE Transactions on Engineering Management, vol.53, n.1, p. 59-67, 2006.

HIKAGE, O. K.; SPINOLA, M. M.; LAURINDO, F. J. B. Software de Balanced Scorecard: proposta de um roteiro de implantação. Revista Produção, v.16, n.1, p. 140-160, 2006.

KIRSH, L. J.; SAMBAMURTHY, V.; KO G.; PURVIS, R. L. Controlling Information Systems Development Projects: The View from the Client. Management Science, vol.48, pp. 484-498, 2002.

MINITAB ${ }^{\circledR}$ Release 14.20 - (C) 1972 - 2005 Minitab Inc. All rights reserved. MINITAB ${ }^{\circledR}$ and the MINITAB $\log { }^{\circledR}$ are registered trademarks of Minitab Inc.

MUSCAT, R. N.; FLEURY, A. C. C. Indicadores da Qualidade e Produtividade na Indústria Brasileira Revista Indicadores da Qualidade e Produtividade - Artigo IV, 2000.

PINTO JK, PRESCOTT JE. Variations in critical success factors over the stages in the project life cycle. Journal of Management 14(1):5-18, 1988.

PINTO JK, SLEVIN DP. Project success: definitions and measurement techniques. Project Management Journal;v.19, n.3, p. 67-73, 1988.

PINTO JR JK, MANTEL SJ. The causes of project failure. IEEE Transactions on Engineering Management, v.37, n.4, p.269-76, 1990.

PINTO,JK; SLEVIN, D.P. Critical factors in successful project implementation. In: FINCH,P. Applying the project implementation profile to an information systems project. Project Management Journal, v.34, n. 3; p. 32, 1987.

PITSIS, T. S.; CLEGG, S. R.; MAROSSZEKY, M.; RURA-POLLEY, T. Constructing the Olympic Dream: A Future Perfect Strategy of Project Management. Organization Science, p. 574-590, 2003.

PMI - PROJECT MANAGEMENT INSTITUTE. A guide to the project management body of knowledge (PMBOK Guide). Upper Darby, PA, 2004.

RAZ, T.; SHENHAR A. J.; DVIR, D. Risk Management, project success, and technological uncertainty. R\&D Management, p. 101-109, 2002. 
SARIN, S.; MAHAJAN, V. The Effect of Reward Structures on the performance of Cross-Functional Product Development Teams. Journal of Marketing, vol.65, p. 35-53, 2001.

SHENHAR, A. J. Strategic Project Leadership. Toward a strategic approach to project management. R\&D Management, p. 569-578, 2004.

STANDISH GROUP. Chaos. Disponível na Internet via WWW. URL: http://www.standishgroup.com Arquivo capturado em 2001.

THAMHAIN, H.J. Linkages of project environment to performance: lessons for team leadership. International Journal of Project Management, v. 22, p. 533-544, 2004.

WEBER, J.; SCHÄFFER, U. On the way to active management of performance measures. International Journal of Business Desempenho Management, p. 47-65, 2001.

YIN, R.K. The case study crisis: some answers. Administrative Science Quarterly. v.26, mar, p.58-65, 1981. 


\section{Anexo}

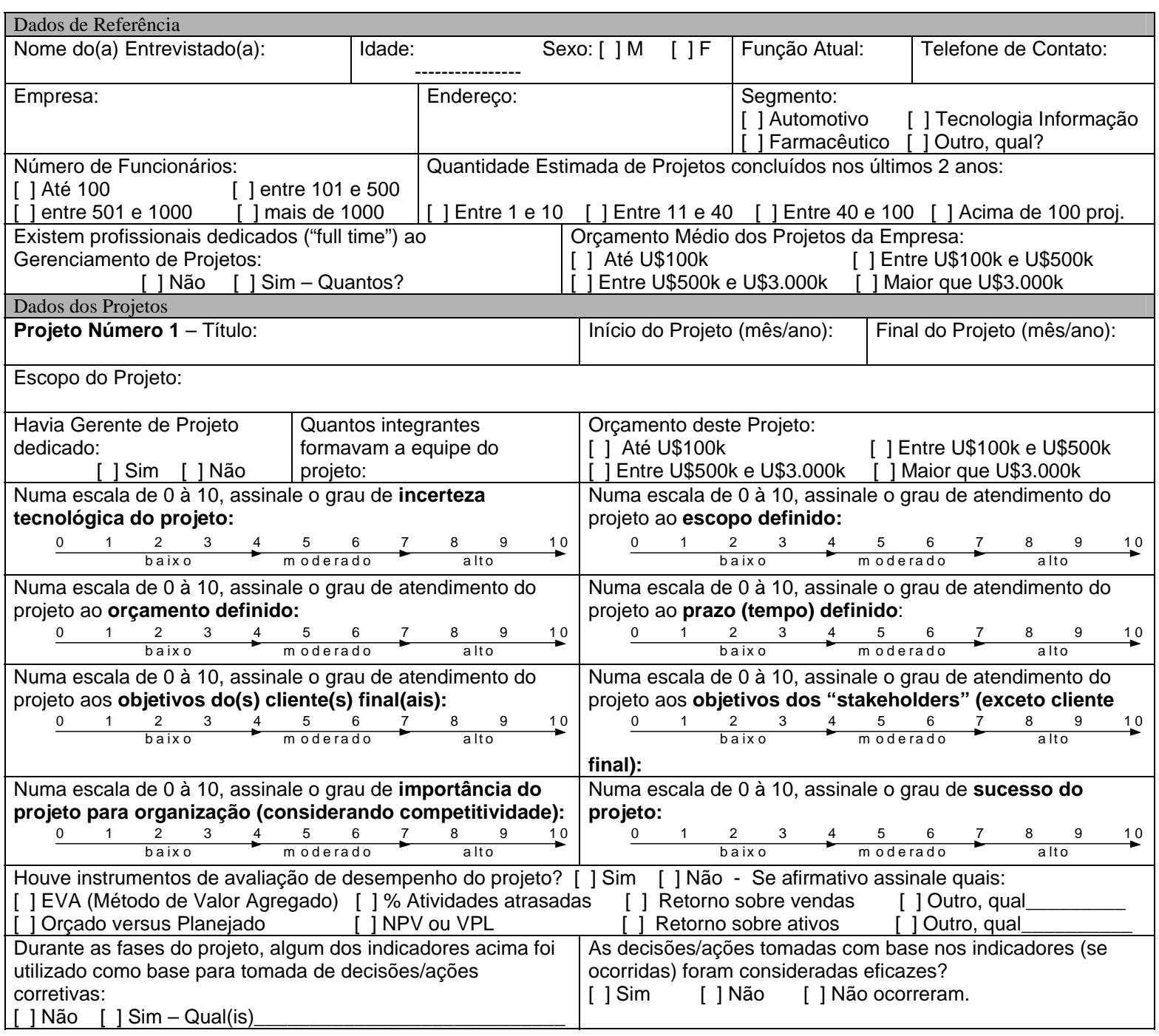

